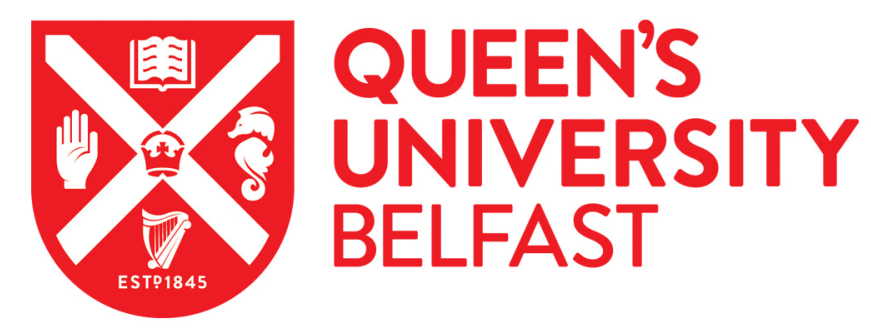

\title{
Anomalous helicon wave absorption and parametric excitation of electrostatic fluctuations in a helicon-produced plasma
}

Krämer, M., Aliev Yu, M., Altukhov, A. B., Gurchenko, A. D., Gusakov, E. Z., \& Niemi, K. (2007). Anomalous helicon wave absorption and parametric excitation of electrostatic fluctuations in a helicon-produced plasma. Plasma Physics and Controlled Fusion, 49(5 A), A167-A175. [S14]. https://doi.org/10.1088/0741$3335 / 49 / 5 \mathrm{~A} / \mathrm{S} 14$

Published in:

Plasma Physics and Controlled Fusion

Queen's University Belfast - Research Portal:

Link to publication record in Queen's University Belfast Research Portal

\section{General rights}

Copyright for the publications made accessible via the Queen's University Belfast Research Portal is retained by the author(s) and / or other copyright owners and it is a condition of accessing these publications that users recognise and abide by the legal requirements associated with these rights.

Take down policy

The Research Portal is Queen's institutional repository that provides access to Queen's research output. Every effort has been made to ensure that content in the Research Portal does not infringe any person's rights, or applicable UK laws. If you discover content in the Research Portal that you believe breaches copyright or violates any law, please contact openaccess@qub.ac.uk. 


\title{
Helicon mode formation and radio frequency power deposition in a helicon-produced plasma
}

\author{
K. Niemi and M. Krämer ${ }^{\mathrm{a})}$ \\ Institut für Experimentalphysik II, Ruhr-Universität Bochum, D-44780 Bochum, Germany
}

(Received 21 December 2007; accepted 29 May 2008; published online 11 July 2008)

\begin{abstract}
Time- and space-resolved magnetic (B-dot) probe measurements in combination with measurements of the plasma parameters were carried out to investigate the relationship between the formation and propagation of helicon modes and the radio frequency ( $\mathrm{rf}$ ) power deposition in the core of a helicon plasma. The Poynting flux and the absorbed power density are deduced from the measured rf magnetic field distribution in amplitude and phase. Special attention is devoted to the helicon absorption under linear and nonlinear conditions. The present investigations are attached to recent observations in which the nonlinear nature of the helicon wave absorption has been demonstrated by showing that the strong absorption of helicon waves is correlated with parametric excitation of electrostatic fluctuations. (C) 2008 American Institute of Physics. [DOI: 10.1063/1.2947561]
\end{abstract}

\section{INTRODUCTION}

It is widely accepted that the strong absorption of helicon waves and the subsequent electron heating in helicon discharges cannot be explained by direct collisional absorption. Since Boswell's pioneering work, ${ }^{1}$ alternative mechanisms based on the electron-wave resonance like Landau damping or nonlinear electron trapping were proposed. ${ }^{2-4}$ Collisionless resonant electron heating may produce hot electrons in the electron energy distribution function. Though, this mechanism may occur at low external magnetic field $\left(B_{0} \leq 10 \mathrm{mT}\right)$ and low plasma density $\left(n_{e} \leq 10^{18} \mathrm{~m}^{-3}\right){ }^{5}$ whereas, probably due to the high collisionality, it is unlikely to play a role in high-density helicon plasma operating in the blue mode. Indeed, in recent investigations on such a plasma, we found no evidence for significant interaction between resonant electrons and helicon waves. ${ }^{6}$

In the present work, we focus on the central region of the helicon discharge that is predominantly affected by helicon propagation and absorption. Thus, we do not consider the region close to the wall under the antenna where other effects (capacitive coupling, mode conversion, etc.) may play a crucial role for the total power balance in helicon discharges as will be seen in Sec. IV. During the last decade linear and nonlinear mechanisms were also discussed, namely, the radio frequency (rf) energy transfer from the helicon wave to other modes either via mode conversion with subsequent absorption or parametric decay of the helicon wave. ${ }^{7-9}$ Recent investigations on a high-density helicon device have shown that the latter mechanism is intimately related to strong helicon wave absorption. ${ }^{10,11}$ Furthermore, the damping of helicon waves can be attributed to a nonlinear process that is closely connected with parametric excitation of short-scale electrostatic fluctuations in the helicon plasma. In some respects, the present study continues previous work. In detail, the helicon wave propagation and mode formation is examined by time- and space-resolved measurements of all mag-

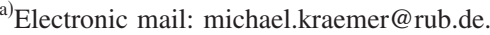

netic components of the helicon wave field in amplitude and phase. Knowledge of the measured rf magnetic field distribution enables us to deduce the Poynting flux and the rf power deposition quantitatively. Applying an $\mathrm{rf}$ double pulse technique, in which the rf power in the second pulse is varied, allows us to study the wave propagation and absorption for different wave amplitudes under nearly identical plasma conditions thus giving further insight in the linear and nonlinear mechanisms of helicon absorption. Another aspect is the effect of the electron density gradient on the helicon propagation and the rf power deposition. Finally, we aim at measuring the power deposited in the core of the helicon plasma in absolute units. This may be important for the selfconsistent description of the helicon discharge.

The paper is organized as follows: In Sec. II, we give a brief outline of the theory of guided helicon waves. In Sec. III, we describe the experimental setup and the diagnostics applied. The experimental results are presented in Sec. IV. Finally, Sec. V contains the conclusions.

\section{THEORY}

\section{A. Linear helicon wave theory}

Helicon waves in magnetized plasma exist in the regime between the lower-hybrid frequency $\omega_{\mathrm{LH}}$ and the electron cyclotron frequency $\omega_{c e}$. If $\omega_{\mathrm{LH}} \ll \omega \ll \omega_{c e}$ and the plasma density is sufficiently high so that $\omega_{p e}^{2} \gg \omega \omega_{c e}$, the propagation of helicon waves can be treated in the framework of the electron MHD (EMHD) theory. [Note that the oblique plasma (Langmuir) wave or Trivelpiece-Gould (TG) wave existing in the helicon regime is not included in this theory.] The governing equations are Maxwell's equations

$$
\begin{aligned}
& \nabla \times \mathbf{E}=-\dot{\mathbf{B}}, \\
& \nabla \times \mathbf{B}=\mu_{0} \mathbf{j},
\end{aligned}
$$

where the vacuum displacement current in Ampere's law (2) is neglected, and Ohm's law 


$$
\mathbf{E}=\eta \mathbf{j}+\frac{1}{e n_{e}} \mathbf{j} \times \mathbf{B}_{0}
$$

resulting from the linearized equation of electron motion in a static external magnetic field $\mathbf{B}_{0}$ when the electron inertia (i.e., the gyromotion) and the ion dynamics are neglected. The plasma resistivity $\eta=\nu_{c} m_{e} / e^{2} n_{e}$ depends on the total collision frequency $\nu_{c}$ that comprises collisional and collisionless loss mechanisms of the electron momentum, namely, electron-ion (ei) and electron-neutral (en) collisions as well as linear electron Landau damping (ELD). The particular collision frequencies read

$$
\begin{aligned}
& \nu_{e i}\left(\mathrm{~s}^{-1}\right)=2.9 \times 10^{-12} n_{e}\left(\mathrm{~m}^{-3}\right)\left[T_{e}(\mathrm{eV})\right]^{-3 / 2} \ln \Lambda_{e} \\
& \nu_{e n}\left(\mathrm{~s}^{-1}\right) \cong 1 \times 10^{7} T_{e}(\mathrm{eV}) p_{\mathrm{Ar}}(\mathrm{Pa}) \\
& \nu_{\mathrm{ELD}}=2 \sqrt{\pi} \omega \zeta^{3} \exp \left(-\zeta^{2}\right)
\end{aligned}
$$

where $T_{e}$ is the electron temperature, $\ln \Lambda_{e}$ is the Coulomb logarithm, $p_{\mathrm{Ar}}$ is the neutral argon gas pressure, and $\zeta$ is the ratio of the wave's phase velocity $v_{\mathrm{ph}}=\omega / k_{z}$ and the thermal electron velocity $v_{e, \mathrm{th}}=\sqrt{2 k_{b} T_{e} / m_{e}}$.

Helicon eigenmodes propagating along a radially inhomogeneous collisionless $(\eta=0)$ magnetized plasma column can be treated by using the wave ansatz $\{\mathbf{E}, \mathbf{B}, \mathbf{j}\}$ $=\{\hat{\mathbf{E}}(r), \hat{\mathbf{B}}(r), \hat{\mathbf{j}}(r)\} \exp \left[\mathrm{i}\left(m \theta+k_{z} z-\omega t\right)\right]$ in Eqs. (1)-(3) to obtain

$$
\nabla \times \mathbf{B}=\alpha\left(\mathbf{B}+i \frac{\beta}{k_{z}} B_{r} \hat{\mathbf{z}}\right),
$$

where

$$
\alpha=\frac{\omega \omega_{p e}^{2}}{\omega_{c e} c^{2} k_{z}}=\mu_{0} e \frac{\omega}{k_{z}} \frac{n_{e}}{B_{0}}, \quad \beta=\frac{d \ln n_{e}}{d r}
$$

is the inverse scale length of the density gradient, and $\hat{\mathbf{z}}$ denotes the unit vector in the $z$ direction.

We briefly describe the procedure to solve Eq. (5) for a given radial density profile (see Refs. 12 and 13). Equation (5) can be decomposed and rearranged leading to a secondorder differential equation for $B_{z}$ with respect to $r$. From its solution expressions for $B_{r}$ and $B_{\theta}$ in terms of $B_{z}$ and $B_{z}^{\prime}$ can readily be obtained. Applying the shooting method the differential equation is numerically integrated (starting from $r$ $=0$ with $B_{z}=1$ and $B_{z}^{\prime}=0$ for $m=0$, and $B_{z}=0$ and $B_{z}^{\prime}=1$ for $m \neq 0$ ) with an initial $k_{z}$ value, which is varied until the corresponding $B_{r}$ solution vanishes at the plasma edge if the plasma is bounded by an ideal conductor. In case of a gap between the plasma boundary and the conducting wall $B_{r}\left(\propto E_{\theta}\right)$ has to vanish at the wall, and $B_{r}$ and $B_{r}^{\prime}$ have to be continuous at the plasma-vacuum boundary. The latter situation is met in our experiment, where the discharge tube is surrounded by a cylindrical metallic screen with $4.5 \mathrm{~cm}$ gap to the plasma boundary. Alternatively, the wave equation can be written as a second-order differential equation for $E_{\theta}$. Here, we start from $r=0$ with $E_{\theta}=0, E_{\theta}^{\prime}=1$ for $m=0$, and $E_{\theta}=1, E_{\theta}^{\prime}=0$ for $m \neq 0$ and vary the (complex) $k_{z}$ until $E_{\theta}$ vanishes at the boundary. In Sec. IV we show numerical results obtained from the latter procedure as it provides more robust computations.

For uniform plasma the total wavenumber is readily obtained from Eq. (5) yielding $k \equiv \sqrt{k_{\perp}^{2}+k_{z}^{2}}=\alpha$ and the dispersion relation

$$
k_{z}=\frac{\omega \omega_{p e}^{2}}{\omega_{c e} c^{2} k} .
$$

The corresponding magnetic field components are then constructed from the Bessel functions $J_{m}\left(k_{\perp} r\right)$ and $J_{m \pm 1}\left(k_{\perp} r\right)$. As the perpendicular wavenumber solely depends on the boundary condition, the width of the magnetic field distribution scales with the plasma diameter, while the axial wavenumber also depends on the plasma parameters. In a radially nonuniform plasma column, there is a cylindrical cutoff layer where the wave characteristic changes from an oscillatory volume-type mode to an evanescent mode.

\section{B. Power deposition by Poynting flux balance}

Wave propagation in a collisional plasma column is formally treated by introducing the complex axial wavenumber $k_{z}=k_{z r}+i k_{z i}$. The real part $k_{z r}$ (the index $r$ will be omitted in the following) has the original physical meaning of $k_{z}$, while the imaginary part $k_{z i}$ denotes the spatial damping decrement. The time-averaged wave energy flux $P$ through an area $A$ is related to the Poynting flux $\mathbf{S}=\mathbf{E} \times \mathbf{B} / \mu_{0}$ by

$$
P=\int_{A}\langle\mathbf{S}\rangle_{t} \cdot \mathrm{d} \mathbf{A}=\frac{1}{4 \mu_{0}} \int_{A}\left(\hat{\mathbf{E}} \times \hat{\mathbf{B}}^{*}+\hat{\mathbf{E}}^{*} \times \hat{\mathbf{B}}\right) \cdot \mathrm{d} \mathbf{A} .
$$

The absorbed wave power $P_{\text {abs }}$ in a certain volume $V$ and the corresponding averaged absorbed wave power density $Q$ can be deduced from the total power flux given by the balance of the inward and outward fluxes through the surface of the volume $A$ according to Poynting's theorem

$$
P_{\mathrm{abs}}=Q \cdot V=-\oint_{A(V)}\langle\mathbf{S}\rangle_{t} \cdot \mathrm{d} \mathbf{A},
$$

where the minus sign accounts for the convention that the normal vector of the surface is directed outwards.

The electrical field vector $\mathbf{E}$ can be expressed in terms of B by using Eqs. (2), (3), and (5) yielding in weakly collisional plasma $(\eta \approx 0)$,

$$
\mathbf{E} \cong \frac{\omega}{k_{z}} \mathbf{B} \times \hat{\mathbf{z}} .
$$

The axial and radial Poynting fluxes then become

$$
\begin{aligned}
& \left\langle S_{z}\right\rangle_{t}=\frac{\omega}{2 \mu_{0} k_{z}}\left(\left|\hat{B}_{r}\right|^{2}+\left|\hat{B}_{\theta}\right|^{2}\right), \\
& \left\langle S_{r}\right\rangle_{t}=\frac{\omega r}{4 \mu_{0} m}\left(\hat{B}_{r} \hat{B}_{\theta}^{*}+\hat{B}_{r}^{*} \hat{B}_{\theta}\right) .
\end{aligned}
$$

In our experiment, the probes are rotated around the axis of the probe shaft thereby intersecting the plasma center at $r=0$. The contributions to the radial and axial fluxes are evaluated separately for both sides $\left(\theta=0^{\circ}\right.$ and $\left.\theta=180^{\circ}\right)$ of 


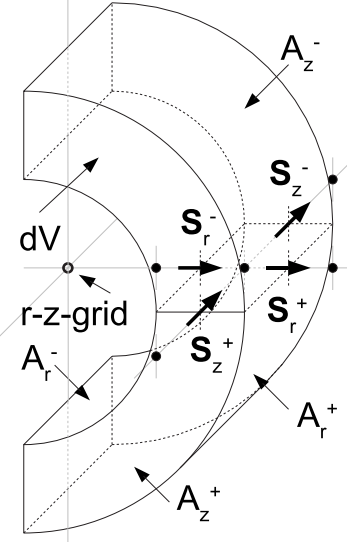

FIG. 1. Control volume geometry.

the axis. In Fig. 1 we have plotted the different fluxes into and out of either cylindrical tube half used in the calculations. Due to symmetry, the azimuthal flux does not, of course, contribute to the wave power deposited. The averaged absorbed power in such a control volume is approximately given by

$$
P_{\mathrm{abs}} \cong S_{z}^{+} A_{z}^{+}-S_{z}^{-} A_{z}^{-}-S_{r}^{+} A_{r}^{+}+S_{r}^{-} A_{r}^{-} .
$$

Note that the (radial) Poynting flux is directed to the axis (implying a positive contribution to the absorbed wave power) and, thus, has negative sign $\left(S_{r}<0\right)$, since the flux is opposite to the radial unity vector $\hat{\mathbf{r}}$.

\section{Power deposition from wave theory}

The locally absorbed wave power density $Q$ is given by the time-averaged scalar product of the current density and the electric field. In the collisional case, $\mathbf{E}$ is no longer perpendicular to $\mathbf{j}$ and $\mathbf{B}_{0}$ as it is easily seen from Eq. (3). Hence, both the parallel and perpendicular components of the current and electric field contribute to the absorbed power. Using Eqs. (2), (3), and (5), we obtain

$$
Q=\langle\mathbf{j} \cdot \mathbf{E}\rangle_{t}=\frac{\eta}{2}|\hat{\mathbf{j}}|^{2}=\frac{\eta}{2 \mu_{0}^{2}}\left|\alpha\left(\hat{\mathbf{B}}+i \frac{\beta}{k_{z}} \hat{B}_{r} \hat{\mathbf{z}}\right)\right|^{2} \text {. }
$$

With the aid of the radial component of Eq. (5) each of the magnetic field components can be expressed by the others. The above equation along with Eq. (9) provides a robust evaluation of the data, inasmuch the singularities of the $1 / r$ terms are avoided and the interfering effect of the $\pi$ phase jumps in the $B_{r}$ and $B_{\theta}$ signals are suppressed when the probes are moved across the center axis due to the reversal of the corresponding unit vectors.

\section{EXPERIMENT}

The investigations were conducted on the pulsed largevolume helicon device HE-L, ${ }^{14}$ shown in Fig. 2. The helicon section consists of a quartz tube $\left(r_{i}=73 \mathrm{~mm}, l=1.1 \mathrm{~m}\right)$ enclosed by a set of 16 coils (inner diameter $=30 \mathrm{~cm}$ ) generating a uniform magnetic field in the axial direction. This section is connected with a large-volume diffusion chamber, which is however not objective of the present work. The helicon plasma is produced by $\mathrm{rf}$ power pulses $(f$ $\left.=13.56 \mathrm{MHz}, P \leq 1.5 \mathrm{~kW}, f_{\text {pulse }}=50 \mathrm{~Hz}, \tau_{\text {pulse }}=2-6 \mathrm{~ms}\right)$ fed to an antenna of $220 \mathrm{~mm}$ length consisting of two flat $180^{\circ}$ turn helical windings with opposite rf current directions surrounding the tube. Such a helical antenna excites predominantly helicon modes with the azimuthal mode numbers $m$ $=+1$ and $m=-1$. The quartz tube and the antenna are enclosed by two aluminum half-shells inside of the magnetic field coils which are connected by copper plates screening the rf fields; the gap between this rf shielding, and the quartz tube amounts to $4.5 \mathrm{~cm}$. Thus, we provide well-defined boundary conditions for the theoretical treatment of helicon mode propagation in this plasma-filled waveguide system, as described in Sec. II.

The typical parameters of the helicon discharge are $n_{e}$ $\leq 10^{19} \mathrm{~m}^{-3}, T_{e} \approx 3 \mathrm{eV}, B_{0} \leq 0.1 \mathrm{~T}, p=0.2-1.0 \mathrm{~Pa}$ for argon. The line-integrated plasma density was measured by a $4 \mathrm{~mm}$ interferometer. Langmuir and rf magnetic (B-dot) probes, which were mounted on a multiprobe holder are movable in the axial and radial direction, enabled local measurements of the plasma parameters and all magnetic components of the helicon wave field.

As sketched in Fig. 3, each of the B-dot probes consists of a small 4-turn coil of $(2 \mathrm{~mm})^{3}$ size. The coils are isolated from the plasma by a glass finger (insulation thickness

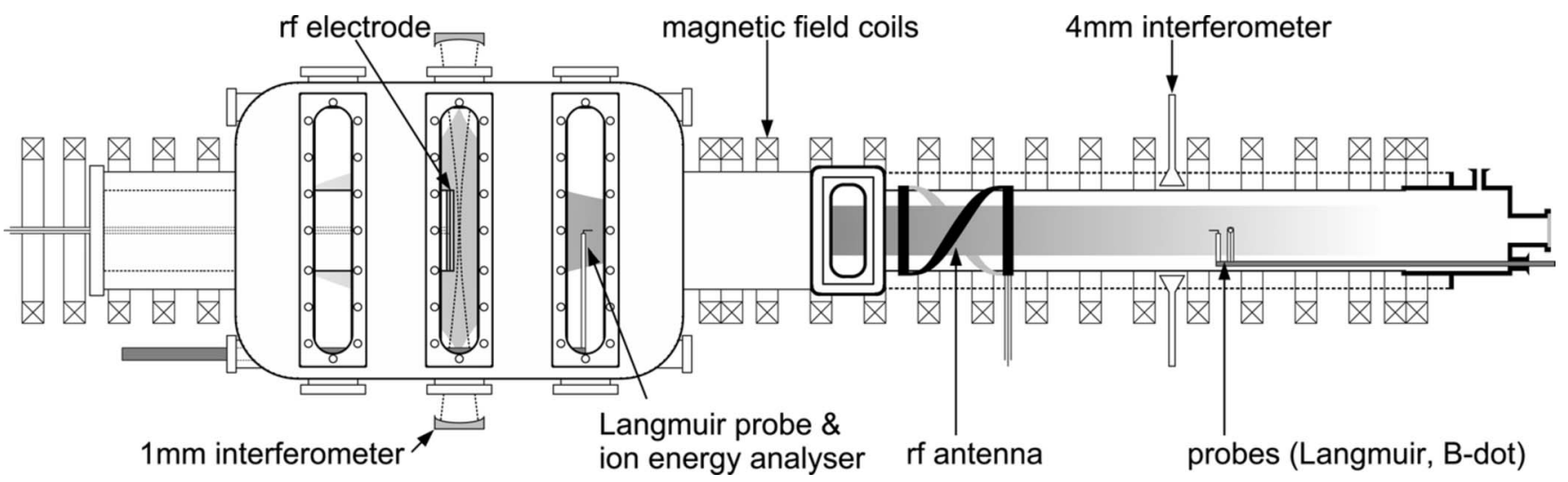

FIG. 2. Setup of the helicon device HE-L. 


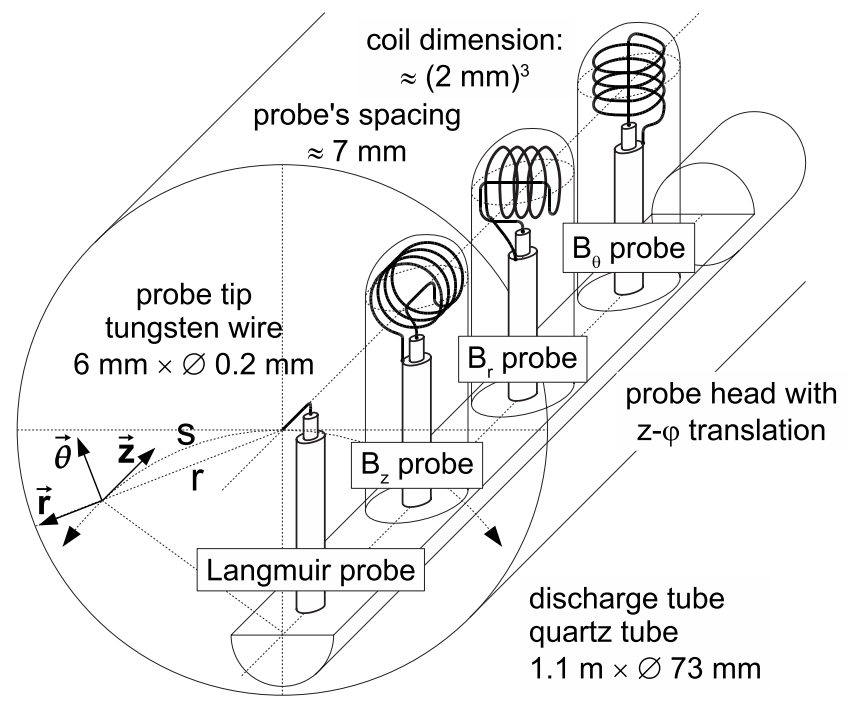

FIG. 3. Schematic of the probe arrangement.

$\approx 0.5 \mathrm{~mm}$ ). The $\mathrm{rf}$ magnetic probe signals were transmitted via $50 \Omega$ coaxial cables of equal length to mixers $\left(R_{i}\right.$ $=50 \Omega$ ) terminating the cables perfectly. To provide full phase and amplitude information, two mixers are needed for each B-dot probe signal; that is, each signal is split in two parts and transmitted to the mixers, where one is mixed in phase, the other $90^{\circ}$ out of phase with a reference signal which comes directly from the small-signal rf generator feeding the rf power amplifier. After low-pass filtering, the output signals are recorded with a digital storage oscilloscope simultaneously with the ion saturation current of the Langmuir probe. In addition, the magnetic probe setup was calibrated by measuring the response of the mixing unit to a defined $\mathrm{rf}$ input, and by comparing the signals with those from an rf magnetic probe of known cross section, i.e., known sensitivity. The probes were also tested with respect to capacitive coupling, as the B-dot probes were not shielded from electrostatic pickup. Knowing the rf test voltage and estimating the rf electric field from helicon wave theory, we were able to deduce the rf voltage at the output of the B-dot probe circuit $\left(R_{i}=50 \Omega\right.$ : input resistance of the mixers; see above). It turned out that this voltage is small (up to a few percent) compared to the induced voltage.

Figure 3 shows that the probe head moves on a circular arc when the multiprobe holder is rotated. In order to reconstruct the helicon wave field in the horizontal midplane of the discharge, the nonlinear scaling of the radial coordinate $r$ with the arc coordinate $s$ has to be observed, the phases of all magnetic probe signals have to be corrected for the actual azimuthal displacement $\Delta \theta$ of the measuring position with respect to the plane by the term $m \Delta \theta$, where the mode number (here, $m=+1$ ) needs to be given, and the amplitudes of the $B_{r}$ and $B_{\theta}$ probe signals have to be decoupled for the actual inclination of both probes with respect to the corresponding unit vectors. The order of the probes with respect to the direction of the wave propagation along $\mathbf{B}_{0}$ (the Langmuir probe is followed by the $B_{z}, B_{r}$, and $B_{\theta}$ probes) was deliberately chosen to avoid mutual screening, because, e.g., the $B_{r}$ or the $B_{\theta}$ probe in front may screen the $B_{z}$ wave field picked up by the adjacent probes. As the measurements agree with preparative measurements performed without the $B_{z}$ and $B_{\theta}$ probe, we were confident that the plasma as well as the helicon wave field is not significantly disturbed by the probes.

We applied an rf double pulse technique in order to study the helicon wave propagation and absorption under linear and nonlinear conditions. The results obtained in the first (main) pulse of $4 \mathrm{~ms}$ duration at $1500 \mathrm{~W}$ generator power were measured under quasistationary discharge conditions, $10 \mu \mathrm{s}$ before turning off the rf power. Then, after a pause of $10 \mu \mathrm{s}$, a second (probe) pulse at reduced rf generator power was launched, and $10 \mu$ s after turning on the rf power again, the second measurement was carried out.

\section{RESULTS}

\section{A. Helicon wave field and helicon dispersion}

An inductive antenna used for launching rf power into a magnetized plasma excites not only helicon waves, but also electrostatic plasma waves. Thus, a considerable part of the rf power may be coupled to the plasma via these Trivelpiece-Gould (TG) waves, which are, however, concentrated near the plasma edge (see, e.g., Ref. 15). The thickness of this outermost region is given by the resonance cone which is defined by the angle of the group velocity with respect to the magnetic field, i.e., $\psi \approx \omega / \omega_{c e}$. For the present conditions, $\omega / 2 \pi=13.56 \mathrm{MHz}$ and $B_{0}=50 \mathrm{mT}$, the resonance cone angle becomes small, $\psi \approx 0.6^{\circ}$, so that the EMHD approximation, which is the basis for evaluating the B-dot probe measurements, is valid in the plasma volume except a thin zone in front of the wall; thus, only helicon wave propagation needs to be considered in this region.

A characteristic feature of helicon discharges with helical antenna coupling is the predominant excitation of $m=+1$ helicon modes travelling in positive magnetic field direction which gives rise to a pronounced axial asymmetry of the rf power deposition with respect to the antenna. ${ }^{15-17}$ As described in Sec. II, guided helicon waves have a nonvanishing wavenumber $k_{\perp}$ perpendicular to the external magnetic field $\mathbf{B}_{0}$ due to the transverse boundary conditions. In the experiment, where the helicon waves are excited via a specific antenna, the situation is more complex as can be seen from Fig. 4 showing a typical quasisnapshot of the amplitude and the phase of the rf magnetic field components in the $r-z$ plane. The helicon wave fronts travel obliquely to the magnetic field from the helical antenna to the center [Fig. 4(b)]. They superimpose there forming a radially standing wave structure that travels along $\mathbf{B}_{0}$ with a well-defined axial wavenumber $k_{z}$. In the center, the field amplitude becomes very large thus leading to maximum rf energy density. The phase diagrams further reveal the structure of the helicon field to become more complex due to superposition of higher radial modes when removing from the antenna. Moreover, the energy profile at high rf power as well as the profiles of the rf magnetic field components exhibit an asymmetry with respect to the axis in that the energy has a maximum nearly at the position of maximum density. Thus, the asymmetry 


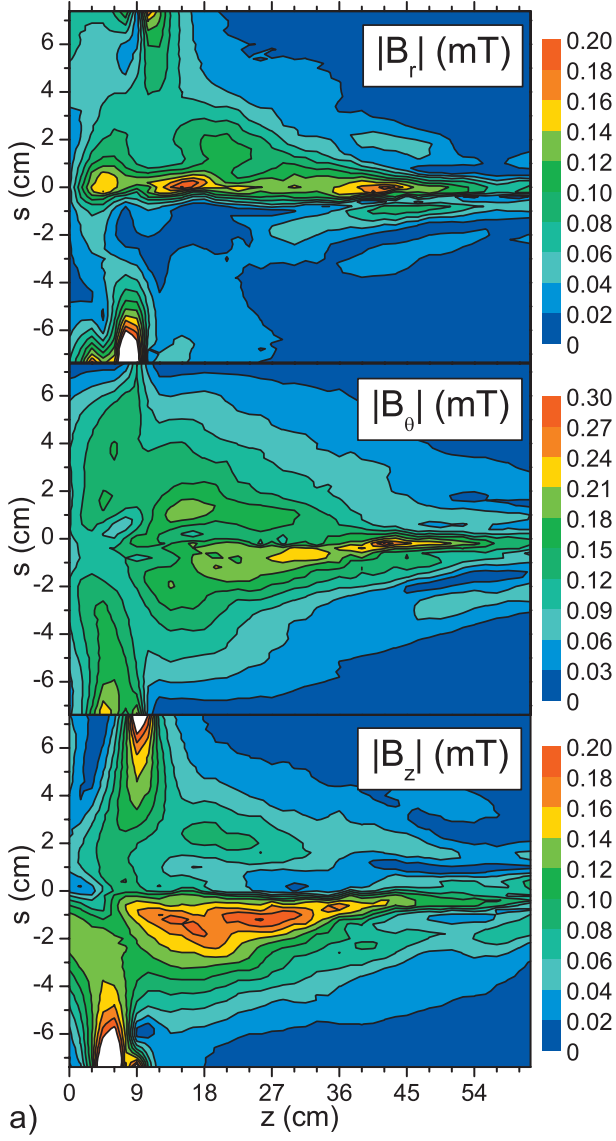

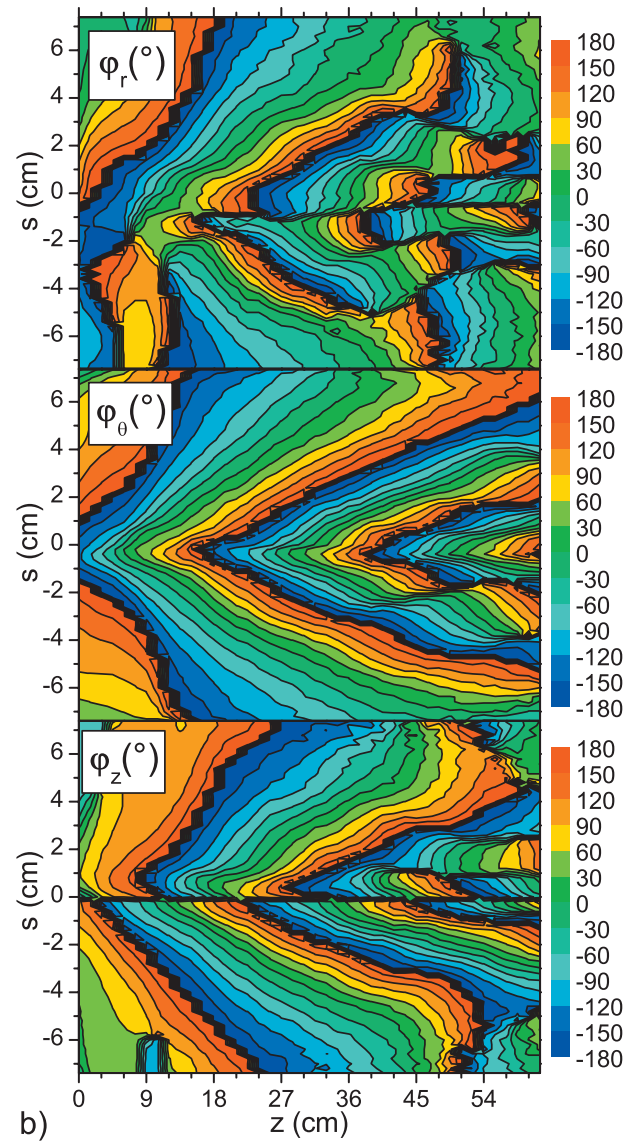

FIG. 4. (Color) Amplitude (a) and phase (b) of the magnetic field components in the quasistationary helicon discharge of the main rf pulse; $B_{0}$ $=50 \mathrm{mT}, p_{\mathrm{Ar}}=0.6 \mathrm{~Pa}, P=1.5 \mathrm{~kW}$. The center of the antenna is located at $z$ $=r=0$. The artificial structures near the antenna winding $(z \approx 9 \mathrm{~cm}$, large $r)$ result from parasitic electrostatic probe pickup. The measured B-dot components reveal continuity $\left(B_{\theta}\right)$ or discontinuity $\left(B_{r}, B_{z}\right)$ on the axis depending on the fact that $B_{z}$ and $B_{\theta}$ change sign and the unity vectors, $\hat{\mathbf{r}}$ and $\hat{\theta}$, are reversed with respect to the $B_{r}$ and $B_{\theta}$ probes, respectively. seems to be correlated with the radially asymmetric density profile. Yet, for low rf power, the rf energy and magnetic field profiles are more symmetric.

One of our main aims was studying helicon wave propagation and absorption under linear and nonlinear conditions. In Fig. 5 we plotted the radial profiles of the electron density and the helicon wave field for high (a) and low (b) launched rf power; the profiles were measured at the axial position $z=40 \mathrm{~cm}$, where the helicon mode has already been formed. At high rf power, the density profiles (top diagrams) are nearly the same except that they have a small narrow peak on top slightly off-axis. Each of the magnetic field components (middle) reveal a broad distribution with a finestructure and a maximum at or close to the center. From these profiles we can easily calculate the rf magnetic energy profiles shown in the bottom diagrams. For high rf power the $|\mathbf{B}|^{2}$ profile turns out to be much narrower than for low power. However, $k_{z}$ remains nearly unchanged although the narrow $|\mathbf{B}|^{2}$ profile at high rf power would indicate a larger $k_{\perp}$ and, thus, a smaller $k_{z}$.

To clarify the above findings we calculated the helicon wave fields with the aid of the helicon waveguide code outlined in Sec. II. We assumed a Lorentzian density profile (plus offset) with and without superposition of a narrow Gaussian of small magnitude which has been fitted to the measured density profiles. The computations shown in Fig. 6 reveal that the small central density peak gives rise to peaked profiles of the perpendicular field components. As a result, the rf energy and the absorbed power density profiles be- come narrow as well, while they are much wider in the "smooth" case of a single Lorentzian. Nevertheless, the axial wavenumber remains nearly unchanged, whereas the rf field and power absorption profiles become significantly narrower which is apparently attributed to the small peak on the electron density profile at high $\mathrm{rf}$ power. This is in qualitative agreement with the observations and cannot be described by the helicon theory for a uniform plasma predicting $k_{z} \propto k_{\perp}^{-1}$ to scale with the width of the $|\mathbf{B}|^{2}$ profile. Obviously, the density gradient term in Eq. (5) plays an important role. Similarly, the small peaks on the absorption profile slightly off axis (Fig. 6) are associated with the gradient term in Eq. (11) for the absorbed power density. (See also the discussion at the end of Sec. IV D.)

In the following, we compare the measurements with theoretical predictions quantitatively. The magnetic field and energy profiles were modeled taking the measured axial wavenumber and assuming a homogeneous plasma column. From the simple dispersion relation (6) we then get the radial wavenumber $k_{\perp} \approx 134 \mathrm{~m}^{-1}$. Alternatively, we estimated the radial wavenumber from the width of the electron density profile yielding an effective plasma radius $a$ and, from the boundary condition $B_{r}(a) \approx 0$, the perpendicular wavenumber $k_{\perp} \approx 100 \mathrm{~m}^{-1}$. In both cases, the resulting profiles of $|\mathbf{B}|^{2}$ are significantly wider than the measured profiles. For low rf power, where the linear theory of helicon waves should be valid, it actually turns out that the ratio between the measured and calculated widths of the radial rf energy profiles amounts to 1.6 and 1.9 , respectively. 


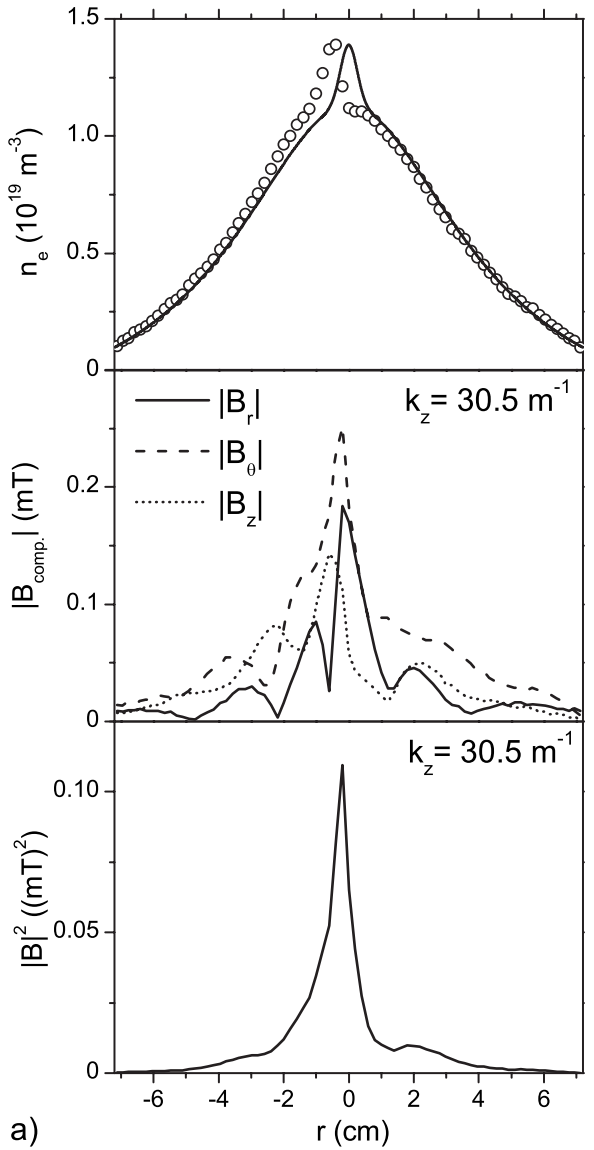

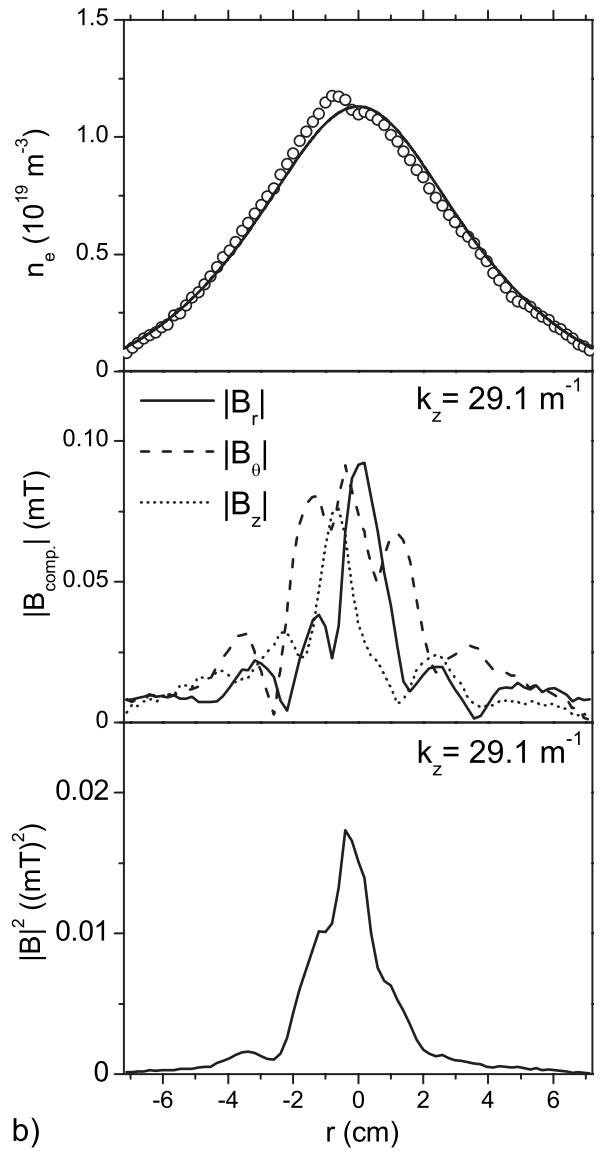

FIG. 5. Measured radial profiles of (top, circles) the electron density, (middle) the magnetic field amplitudes, and (bottom) the rf energy density for high (a) and low (b) launched rf generator powers, $P=1.5 \mathrm{~kW}$ and $P=100 \mathrm{~W}$, respectively. Measuring position $40 \mathrm{~cm}$ away from the center of the antenna, $B_{0}=50 \mathrm{mT}, p_{\mathrm{Ar}}$ $=0.6 \mathrm{~Pa}$; solid lines in top diagrams used for modelling.
The discrepancy between the measurements and the theoretical predictions persists even if we take account of the finite axial extension of the helicon field thus leading to a corresponding axial wavenumber spectrum. Moreover, the modelled profile of the magnetic energy is smooth in contrast to the measured profiles revealing a fine structure and, thus, suggesting the existence of higher radial modes (see Fig. 5, middle). According to helicon theory, larger radial mode numbers would imply smaller values of $k_{z}$ so that one would expect a spectrum of axial wavenumbers. Computations for the probe pulse using our EMHD waveguide code yield that the helicon modes with the radial mode numbers $1,2,3,4, \ldots$, i.e., the number of nodes of $B_{r}$, have the axial wavenumbers $k_{z}\left(\mathrm{~m}^{-1}\right)=47.6,28.1,18.8,14.0, \ldots, \quad$ respectively. However, this contrasts with the measured helicon wave field exhibiting essentially only one axial wavenumber.

\section{B. RF power deposition}

The rf power deposited in the helicon plasma column is given by the energy flux balance. We determined the radial and axial components of the Poynting flux vector, $S_{r}$ and $S_{z}$, using Eq. (10). In Fig. 7 we plotted the axial Poynting flux, which is large compared to the radial flux. With regard to the helicon waveguide model used in Sec. IV A it is worth noting that the angle of the energy flux with respect to the magnetic field direction tends to zero although from the wave fronts shown in Fig. 4(b) and the helicon dispersion relation we would deduce an angle of the group velocity amounting to about $10^{\circ}$. Hence, this model should describe the observed helicon modes sufficiently well. Since $S_{z}$ is proportional to $\left|B_{\perp}\right|^{2}$, it exhibits a similar structure as the measured rf energy density. Considering the radial flux, it turns out that the probes cannot properly resolve the zeroes of the corresponding components in the relevant $B_{r} B_{\theta}^{*}$ term in Eq. (10b), because the probe coil has finite size $\left(\approx 2 \mathrm{~mm}^{3}\right)$ and picks up undesired components $(5 \%-10 \%)$. Moreover, when the $B_{r}$ and $B_{\theta}$ probes are moved across the axis, their signals undergo a phase jump of $\pi$ since the corresponding unit vectors reverse. As a result (not shown), the calculation of the $\mathrm{rf}$ power deposition gives rise to artificial structures, particularly in the vicinity of the axis. Nonetheless, the absorbed power deduced from Eq. (11) for the main volume taken in the range $z \geq 13 \mathrm{~cm}$ yields reliable values. For high $\mathrm{rf}$ power (main pulse) we obtained the total absorbed power $P_{\text {abs }, h}$ $=313 \mathrm{~W}$, while $P_{\mathrm{abs}, l}=40 \mathrm{~W}$ for low rf power (probe pulse). The ratio of the absorbed powers, $R_{\mathrm{abs}, h}=P_{\mathrm{abs}, h} / P_{a b s, l} \approx 8$, nearly corresponds to the ratio of the maximum rf field energies of about 7. Comparing the absorbed rf powers with the powers $P_{\mathrm{rf}, h}=1500 \mathrm{~W}$ and $P_{\mathrm{rf}, l}=100 \mathrm{~W}$, respectively, launched to the plasma, we see that $P_{\mathrm{abs}, h}$ and $P_{\mathrm{abs}, l}$ are considerably smaller. Obviously, the major portion of the $\mathrm{rf}$ power is absorbed in the outer regions of the plasma, most likely close to the antenna. This holds in particular for high rf power, where the power deposited in the helicon-sustained plasma amounts only to about $300 \mathrm{~W}$ corresponding to $20 \%$, while the relative power deposited in the central region is nearly twice higher for low rf power coupling. This indicates 

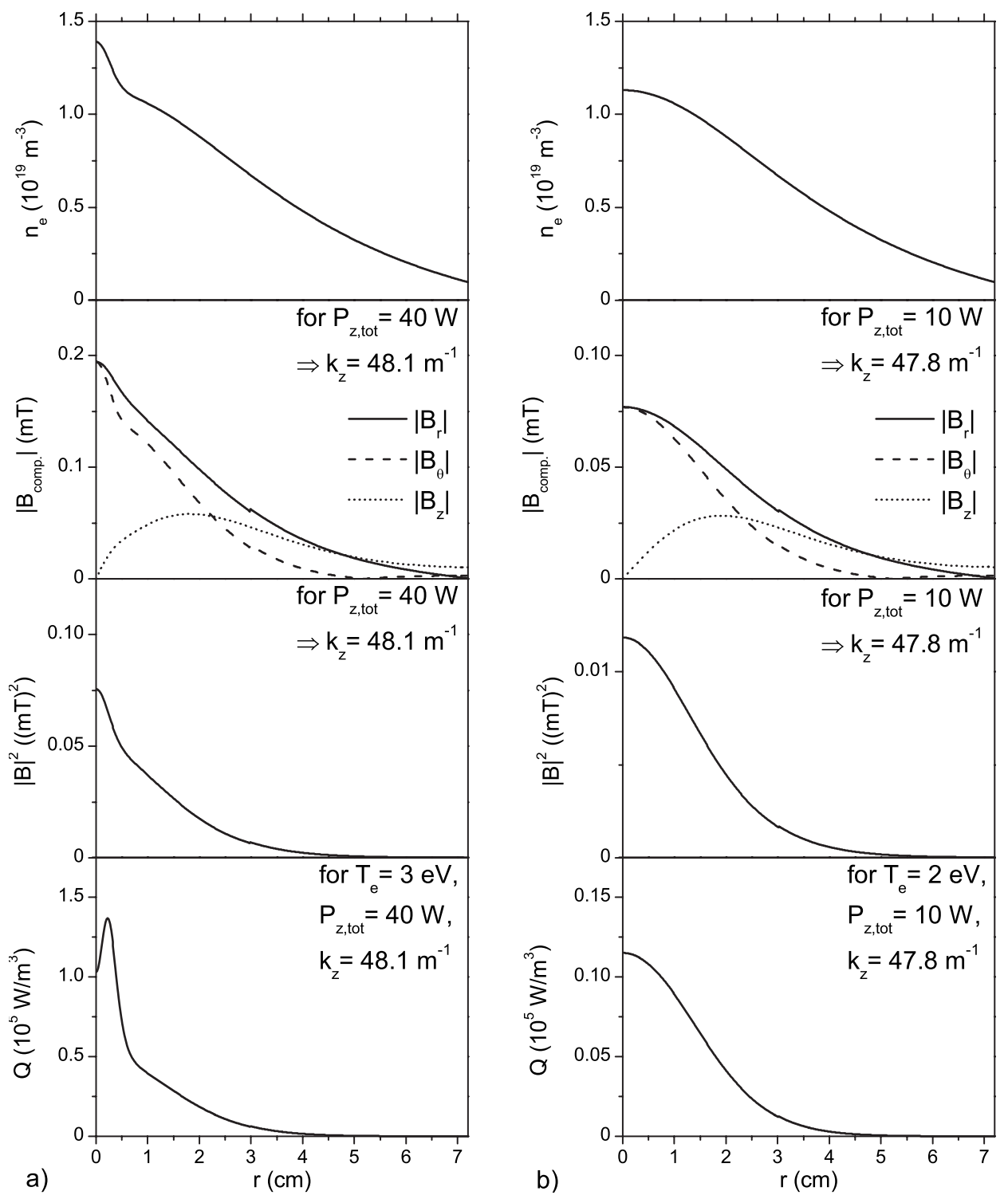

FIG. 6. Computation of the radial field distribution and the absorbed power density for different model density profiles (adapted to the measured profiles).

that the rf absorption near the antenna is, at least in part, nonlinear, inasmuch amplitude-dependent processes occur.

Our measurements allow us to decide whether the rf power absorption in the helicon region can be attributed to a linear or a nonlinear mechanism. We compared the above results with the absorbed power that is deduced from the rf field distribution making use of the helicon wave theory for a collisional plasma (Sec. II). As is seen from Fig. 8 (top), the rf power deposition as well as the measured rf field energy density for high power are more concentrated on the axis than for low power. This has been predicted by the nonuniform EMHD model which takes account of density gradient effects via the $\beta$ term in Eq. (11). To evaluate the measurements we integrated the absorbed power density over the volume yielding the total absorbed powers $P_{c a, h}=40.1 \mathrm{~W}$ for high $\mathrm{rf}$ power and $P_{c a, l}=11.7 \mathrm{~W}$ for low power resulting in the ratio $P_{c a, h} / P_{c a, l}=3.4$. In particular, the power $P_{c a, h}$ is by far too low compared to the measured value $P_{\mathrm{abs}, h}=313 \mathrm{~W}$
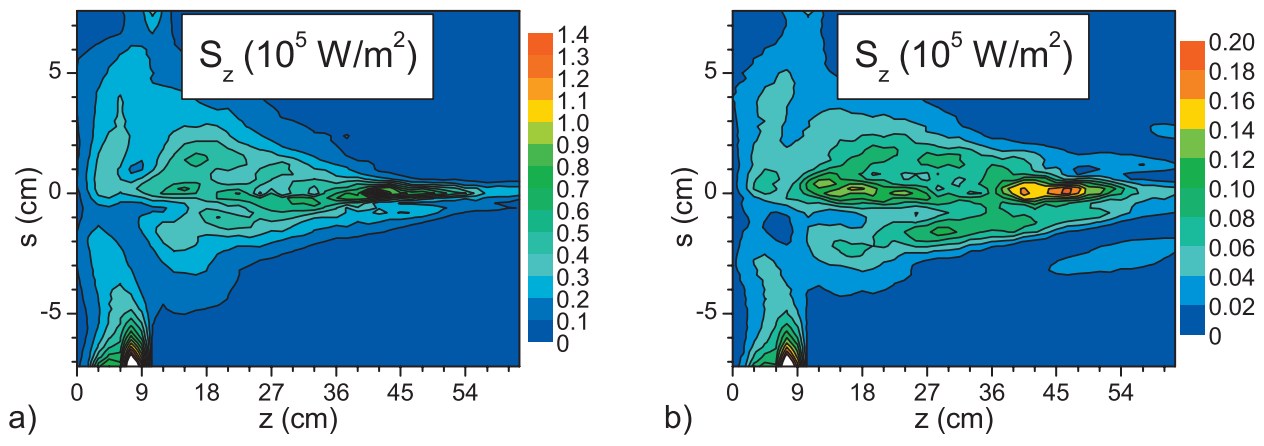

FIG. 7. (Color) Axial Poynting flux for high (a) and low (b) launched rf generator powers, $P=1.5 \mathrm{~kW}$ and $P$ $=100 \mathrm{~W}$, respectively. $B_{0}=50 \mathrm{mT}$, $p_{\mathrm{Ar}}=0.6 \mathrm{~Pa}$. 

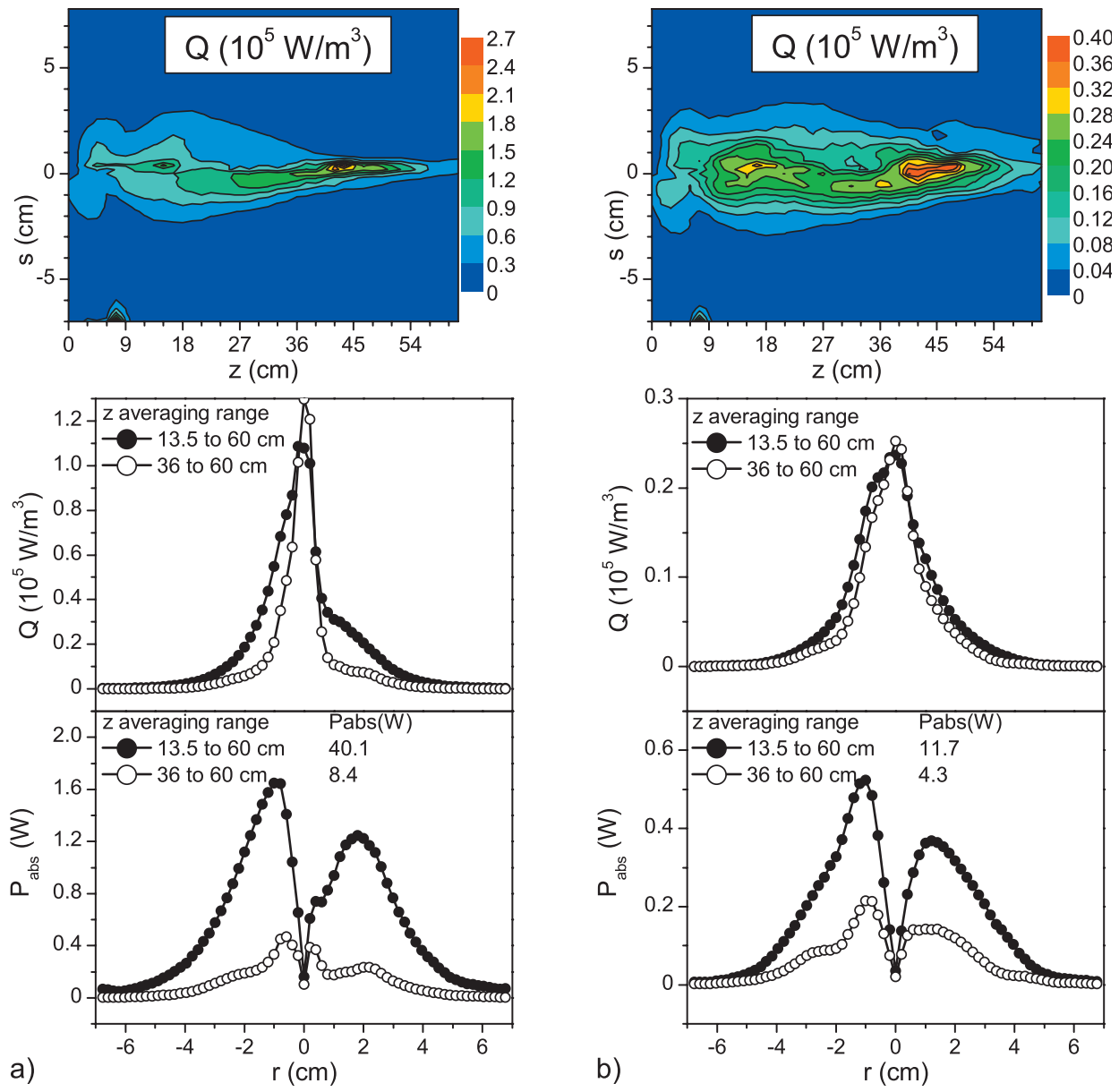

FIG. 8. (Color) (Top) 2D plot of the absorbed power density (a) in the main pulse $\left(k_{z}=30.5 \mathrm{~m}^{-1}, T_{e}=3 \mathrm{eV}\right)$ and (b) in the probe pulse $\left(k_{z}=29.1 \mathrm{~m}^{-1}, T_{e}\right.$ $=2 \mathrm{eV})$; (middle) radial profiles of the absorbed power density averaged over the $z$ intervals indicated; (bottom) resulting contributions to the absorbed power.

thus indicating that an anomalous absorption mechanism is involved. Even in case of low rf power, electron-ion collisions, electron-neutral collisions, and electron Landau damping are not sufficient to account for the power absorption. A linear mechanism, which may, in principle, play a role in the central region of the plasma column is mode conversion of helicon waves into Trivelpiece-Gould waves. This mechanism occurs, if the plasma density in the conversion zone $\left(r=r_{m c}\right)$, defined by the relation $N_{z}=2 \omega_{p e}\left(r_{m c}\right) / \omega_{c e}{ }^{7}$ is exceeded. However, for the axial wavelength $\lambda_{z} \approx 22 \mathrm{~cm}$, the corresponding density becomes $n_{e}\left(r_{m c}\right) \approx 6.4 \times 10^{19} \mathrm{~m}^{-3}$ which is much higher than the central density in our discharge. Nevertheless, theoretical investigations carried out very recently show that the conversion zone in strongly nonuniform plasma can shift to much lower densities. ${ }^{18}$ Indeed, the density gradient associated with the peak near the axis occurring at high power is steep enough to fulfill the new conversion condition given in Ref. 18, whereas this does not hold for the relatively smooth profile at small power. Further theoretical work on helicon absorption in nonuniform plasma has been done by Breizman and Arefiev ${ }^{19}$ who predicted radially localized helicon modes which may lead to efficient rf absorption. However, they are unlikely to occur for our conditions.

In order to clarify how the different regions in the helicon-sustained plasma contribute to the total power absorption we compared in Fig. 8 (middle and bottom) the absorbed power densities averaged over the $z$ intervals 13.5 $\leq z / \mathrm{cm} \leq 60$ and $36 \leq z / \mathrm{cm} \leq 60$ with the power deposited in cylindrical shells of corresponding length. It can be seen that
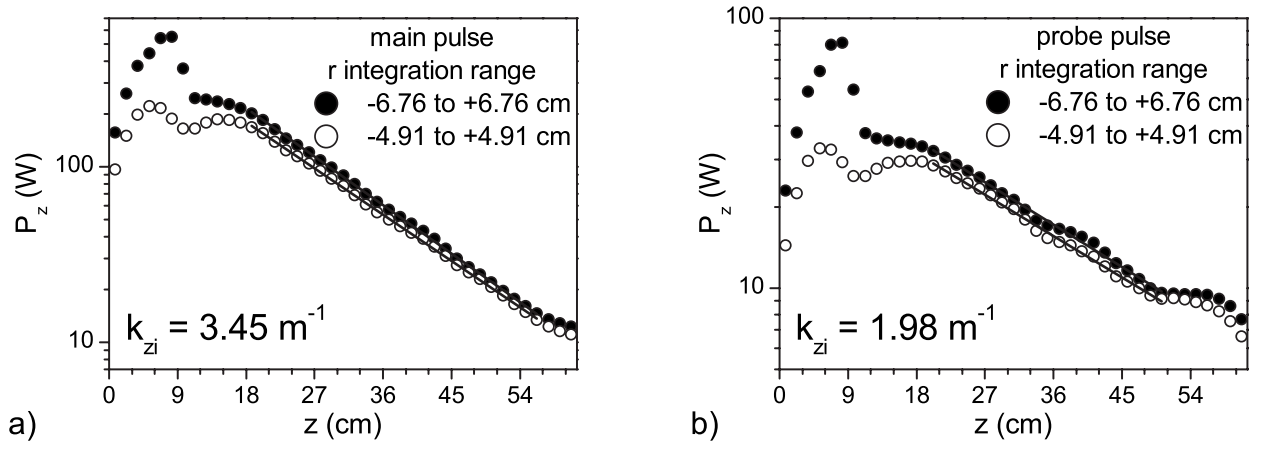

FIG. 9. Axial wave energy flux (integrated over the cross section indicated) for high (a) and low (b) rf power. 
the main contribution to the power absorption comes from the region that is about $1.5-2.0 \mathrm{~cm}$ away from the axis. Due to cylindrical geometry, the region near the axis thus yields only a small contribution, although the power absorption (per unit volume) has a maximum there. Hence, this region plays only a minor role for the total power absorption, but the absorption mechanism(s) being active there are, of course, decisive for the formation of the density profile near the axis.

\section{Dependence of the helicon damping on the rf power}

Further insight in the helicon absorption can be gained from the axial dependence of the Poynting flux, from which the axial damping decrement of the helicon modes can be deduced. This has already been objective in previous investigations of helicon wave damping where we made use of the fact, that the energy flux along the plasma column is exponentially damped. ${ }^{10}$ Integrating the Poynting flux $S_{z}$ over the cross section we obtain the damping decrement $k_{z i}$ from the spatial dependence $\exp \left(-2 k_{z i} z\right)$. For high rf power, $k_{z i}$ turns out to be considerably larger than expected for collisional damping as it is seen in Fig. 9. In that paper, we also observed that the helicon damping and the level of the lf and $\mathrm{hf}$ electrostatic fluctuations increases with the $\mathrm{rf}$ power. This suggests the intimate relationship between the helicon absorption and these fluctuations which were evidenced to be excited by the helicon wave field via a parametric instability. To examine the dependence of the helicon damping on the rf power and the excitation of the fluctuations in more detail we applied a more refined rf pulse technique. The first (main) $\mathrm{rf}$ pulse generating the helicon plasma is followed by a second pulse with a descending step (staircase) structure. In Fig. 10(a), the rf power has been plotted along with the electron temperature and the plasma density. As it is seen, $T_{e}$ decreases significantly while $n_{e}$ stays nearly constant. As a result (not shown), the helicon wavelength remains nearly unchanged as well; from Fig. 4(b) we deduce $\lambda_{z r}=0.215 \mathrm{~m}$ or $k_{z r}=29.2 \mathrm{~m}^{-1}$. Figure 10(b) shows the dependence of the $\mathrm{rf}$ magnetic energy density in the center as well as the energy flux through the plasma cross section. Two opposite effects apparently occur, (i) the focusing of the helicon field close to the axis (at lower rf powers, $|\mathbf{B}|^{2}$ increases more strongly than proportionally) and (ii) the decrease of the energy flux relative to the launched rf power which may be attributed to the enhanced absorption under the antenna. Finally, in Fig. 10(c), the dependence of the helicon damping on the $\mathrm{rf}$ power has been plotted. The damping rate starts growing at a certain rf power, which approximately coincides with the power at which the fluctuations set in. Our observations thus confirm that the helicon damping reveals a threshold which is characteristic for parametric excitation of electrostatic fluctuations.

At low rf power, the measured damping rates are expected to depend primarily on electron collisions while electron Landau damping can be neglected. The predicted damping rates turn out to be nearly constant, but they are almost three times smaller than the measured rates at low rf powers. This is quantitatively consistent with the rf power deposition
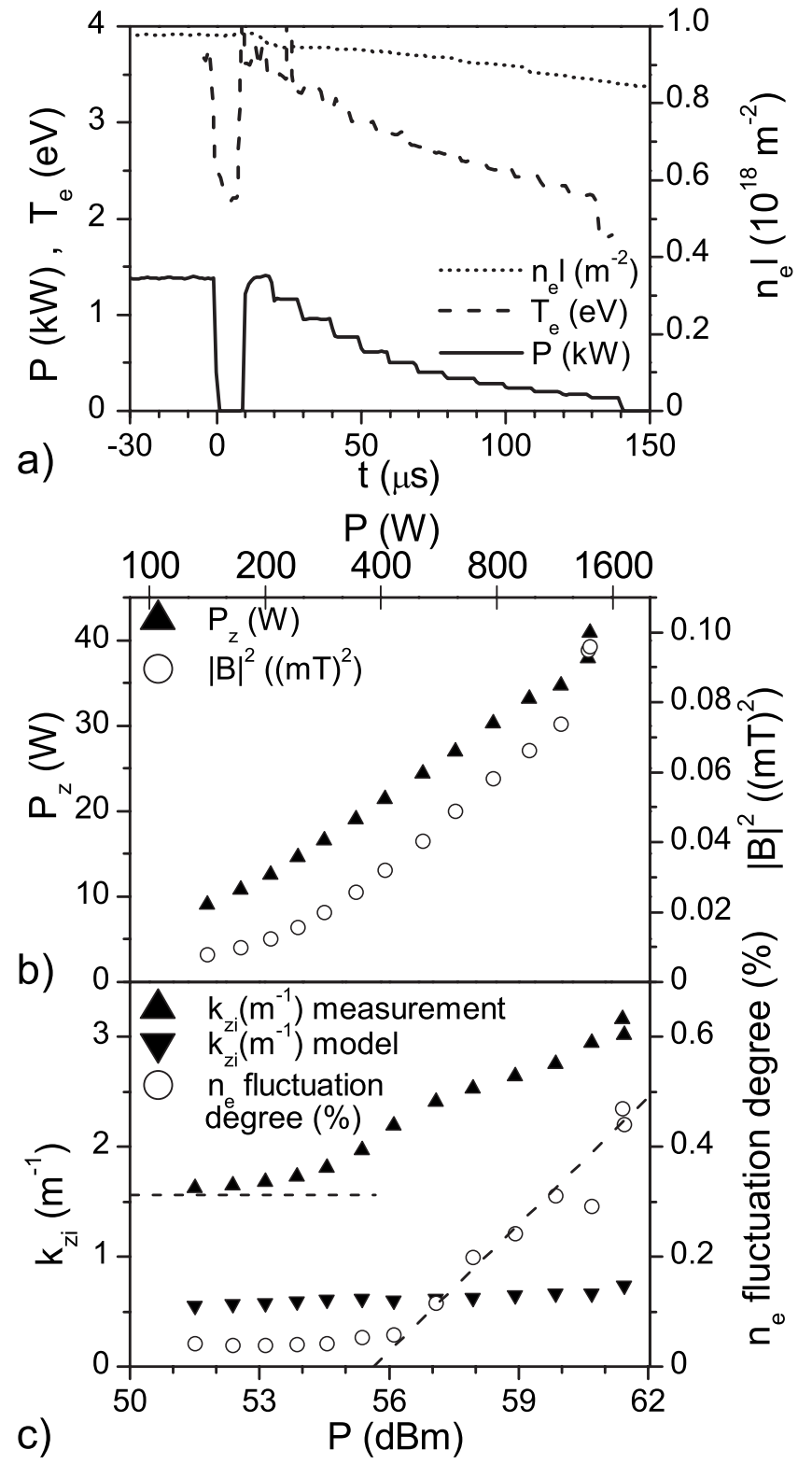

FIG. 10. (a) Evolution of electron temperature $T_{e}$ and line-of-sight integrated electron density $n_{e} l$ from the end of the main rf pulse to the end of the rf probe pulse. (b) Axial power flux $P_{z}$ and on-axis rf magnetic field and (c) damping decrement $k_{z i}$ and degree of electron density fluctuations in the frequency range from 0.3 to $5 \mathrm{MHz}$ vs rf power. Measuring position $40 \mathrm{~cm}$ away from the center of the antenna, $B_{0}=50 \mathrm{mT}, p_{\mathrm{Ar}}=0.6 \mathrm{~Pa}$.

which was also observed to be considerably larger than expected for collisional absorption (see Sec. IV B). Indeed, the ratio of the absorbed power measured for low rf power, $P_{\mathrm{abs}, l}=40 \mathrm{~W}$ and the power calculated from the rf energy assuming only collisional absorption $P_{c a, l}=11.7 \mathrm{~W}$ yielding $P_{\mathrm{abs}, l} / P_{c a, l}=3.4$, which is in reasonable agreement with the measured and calculated damping decrements.

\section{Electron temperature and density distributions}

In Sec. IV B we have seen that the rf power is deposited in the center via helicon modes and, even the major part, in the region close to the antenna. Therefore, we expect the electron heating and plasma production primarily in these 


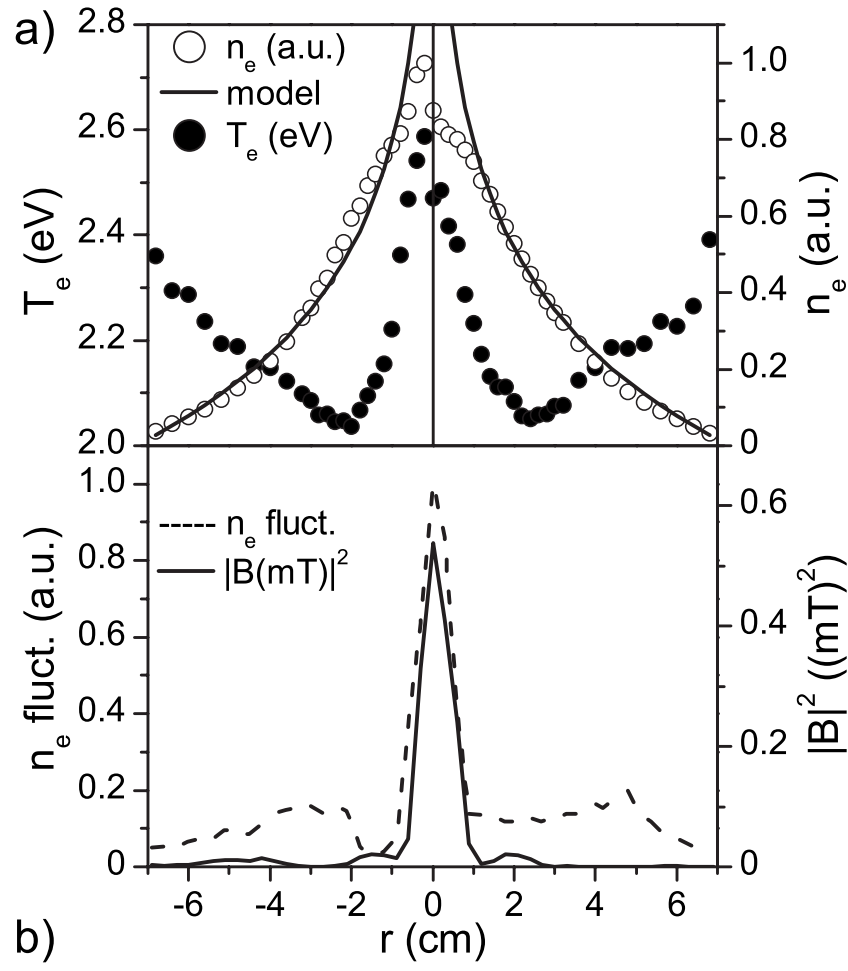

FIG. 11. (a) Radial profiles of electron temperature $T_{e}$ and density $n_{e}$; the solid line results from a simple diffusion model assuming a line source on axis. (b) Radial profiles of electrostatic fluctuation amplitude (0.3-5 MHz) and rf magnetic energy density $\propto|B|^{2}$. Measuring position $49 \mathrm{~cm}$ away from the center of the antenna, $P=1000 \mathrm{~W}, B_{0}=55 \mathrm{mT}, p_{\mathrm{Ar}}=0.3 \mathrm{~Pa}$.

regions. As it is seen in Fig. 11(a), the profile of the electron temperature $T_{e}$ peaks on the axis, and grows again towards the plasma edge. (Note that these measurements were carried out for somewhat different conditions.) Apparently, the rf power deposited near the antenna causes heating of electrons, which can still be detected far away from the antenna due to high thermal conductivity along the magnetic field. Unlike the temperature profile, the electron density exhibits a much broader profile due to cross-field diffusion (see below). Moreover, contrary the temperature distribution, the decrease of the density towards the plasma edge can probably be attributed to strong particle losses taking place mainly at the wall under the antenna.

We now disregard the latter region and focus on the central region which is the main objective of this paper. In Fig. 11(b), radial profiles of the rf magnetic energy and the level of the electrostatic fluctuations have been plotted. It turns out that the rf energy as well as the fluctuation level are both concentrated near the axis proving again their intimate relationship (see Sec. IV C). The rf absorption and the electron heating are therefore expected to have pronounced maxima in the center. In order to understand the density distribution, let us consider the simple model of a line plasma source at the plasma axis described by a delta functional ionization frequency. We then obtain the radial dependence $n_{e}(r) \propto \ln \left(r_{p} / r\right)$ [solid line in Fig. 11(a)] fitting well with the measured density profile, if the singularity at the axis is ignored. This model is, of course, very simple and does not account for the processes taking place near the axis, but it confirms our assumption that the density profile is governed by plasma production mainly close to the axis and cross-field diffusion. (More comprehensive investigations of the helicon discharge physics have been carried out in Ref. 6.)

In the end, we discuss an interesting feature of the electron density profile, that is, the small peak on top observed slightly off axis. A qualitative explanation of this finding may be as follows. Let us start with a narrow axisymmetric density profile. According to Fig. 6(a) (bottom) the absorption is expected to have its maximum off axis if the density gradient is steep enough. As a result, there is an annular zone of maximum absorption. Let us now suppose a slight deviation from axisymmetry so that the absorption is somewhat stronger at a certain point of the ring. The electron heating and the plasma production at this point is then slightly stronger thus leading to further rise of the absorption and so forth. We thus expect the annular absorption zone to be unstable. Clearly, the formation of the helicon-sustained discharge is a very complex process, inasmuch the plasma density profile establishes in a self-consistent manner during the evolution of the discharge.

\section{CONCLUSIONS}

Detailed measurements of the helicon wave fields and the plasma parameters in two dimensions have been carried out using an arrangement of three B-dot probes and one Langmuir probe. The time- and space-resolved measurements of all rf magnetic field components in amplitude and phase enables us to deduce the rf power deposition in the helicon plasma quantitatively. A particular issue of our investigations was the comparison of the helicon mode formation and propagation as well as the rf energy flux and power deposition under linear and nonlinear conditions, i.e., for low and high rf power coupling. Strong focusing of the helicon wave energy was observed at high rf power, which can partly be attributed to the peaked radial electron density profiles. However, the comparison with computations based on a helicon waveguide model reveals that the measured profiles of the rf field energy are narrower than the profiles computed with the aid of the measured axial wavelength. Alternatively, we can say that the wavelength is expected to be longer if the radial rf energy profiles are taken in the calculations.

For the first time, the rf power deposited in the helicon discharge via helicon modes has been determined in absolute units; this provides a useful benchmark for the proper modelling of helicon discharges. In the case of maximum rf power coupling with $P_{\mathrm{rf}}=1.5 \mathrm{~kW}$, the deposited power amounts only to about $300 \mathrm{~W}$, while the relative power is nearly twice as high for low rf power coupling. In both cases, the power deposited in the plasma via helicon waves are much smaller than the total power launched to the plasma. Hence, the rf power is primarily absorbed in the outermost regions of the plasma near the antenna. The difference between the relative powers for low and high power coupling deposited in the plasma core indicates that the rf absorption near the antenna is, at least in part, a nonlinear process. 
For low and high rf power coupling, the measured powers deposited in the plasma via helicon waves are significantly higher than inferred from the rf magnetic fields if only electron collisions are taken into account. These findings are in accordance with measurements of helicon damping. Even at small wave amplitude, the observed damping turns out to be about three times stronger than predicted from helicon theory for collisional plasma. When increasing the rf power, the helicon damping starts increasing above a certain level thus indicating a nonlinear absorption mechanism. This threshold behavior is consistent with the existence of a parametric instability, which is also supported by the electrostatic fluctuations mainly observed in the central region, where the rf energy is largest. It is therefore likely that dissipation via parametrically excited electrostatic fluctuations plays an important role in the helicon discharge. Other mechanisms associated with the steep density gradient (modified mode conversion) and/or the electron-wave resonance may come into play close to the axis of the plasma column. Such processes may play a role for the plasma production and the formation of the density distribution near the axis. However, they are less important for the total power absorption since they are efficient only in a small volume.

\section{ACKNOWLEDGMENTS}

The authors thank Yu. M. Aliev for valuable discussions. The technical assistance of M. Konkowski is highly appreciated.
This work was supported by the Deutsche Forschungsgemeinschaft through the Sonderforschungsbereich 591.

${ }^{1}$ R. W. Boswell, Plasma Phys. Controlled Fusion 26, 1147 (1984).

${ }^{2}$ F. F. Chen, Plasma Phys. Controlled Fusion 33, 339 (1991).

${ }^{3}$ A. R. Molvik, A. R. Ellingboe, and T. D. Rognlien, Phys. Rev. Lett. 79, 233 (1997).

${ }^{4}$ A. R. Ellingboe, R. W. Boswell, J. P. Booth, and N. Sadeghi, Phys. Plasmas 2, 1807 (1995).

${ }^{5}$ S. M. Tysk, C. M. Denning, J. E. Scharer, and K. Akhtar, Phys. Plasmas 11, 878 (2004).

${ }^{6}$ B. Clarenbach, M. Krämer, and B. Lorenz, J. Phys. D 40, 5117 (2007).

${ }^{7}$ K. P. Shamrai and V. P. Taranov, Plasma Sources Sci. Technol. 5, 474 (1996).

${ }^{8}$ A. I. Akhiezer, V. S. Mikhailenko, and K. N. Stepanov, Phys. Lett. A A245, 117 (1998).

${ }^{9}$ Yu. M. Aliev and M. Krämer, Phys. Plasmas 12, 072305 (2005).

${ }^{10}$ B. Lorenz, M. Krämer, V. L. Selenin, and Yu. M. Aliev, Plasma Sources Sci. Technol. 14, 623 (2005).

${ }^{11}$ M. Krämer, Yu. M. Aliev, A. B. Altukhov, A. D. Gurchenko, E. Z. Gusakov, and K. Niemi, Plasma Phys. Controlled Fusion 49, A167 (2007).

${ }^{12}$ M. Krämer, Phys. Plasmas 6, 1052 (1999).

${ }^{13}$ F. F. Chen, M. J. Hsieh, and M. Light, Plasma Sources Sci. Technol. 3, 49 (1994).

${ }^{14}$ M. Krämer, B. Lorenz, and B. Clarenbach, Plasma Sources Sci. Technol. $11 \mathrm{~A}, 120$ (2002).

${ }^{15}$ T. Enk and M. Krämer, Phys. Plasmas 7, 4308 (2000).

${ }^{16}$ S. Shinohara, Y. Miyauchi, and Y. Kawai, Plasma Phys. Controlled Fusion 37, 1015 (1995).

${ }^{17}$ I. D. Sudit and F. F. Chen, Plasma Sources Sci. Technol. 5, 43 (1996).

${ }^{18}$ Yu. M. Aliev and M. Krämer, "Mode conversion of nonaxisymmetric modes in strongly nonuniform helicon-sustained plasma," Phys. Plasmas (submitted).

${ }^{19}$ B. N. Breizman and A. V. Arefiev, Phys. Rev. Lett. 84, 3863 (2000). 